\title{
AS TÉCNICAS NASVD E MNF E SUA APLICAÇÃO NA REDUÇÃO DE RUÍDOS EM DADOS GAMAESPECTROMÉTRICOS
}

\author{
Francisco de Assis Cavallaro ${ }^{1,4}$, Rodrigo S. Portugal ${ }^{2}$, Adalene M. Silva ${ }^{3}$ e Ariathemis M. Bizuti² \\ Recebido em 22 julho, 2008 / Aceito em 15 maio, 2009 \\ Received on July 22, 2008 / Accepted on May 15, 2009
}

\begin{abstract}
The radioactive decay is a random process, and the measurement precision is ruled by statistical laws. The counting ratios of the profiles are always noisy when analyzed for short periods, such as one second per measurement. Corrections made at the end of conventional processing in the airborne gamma-ray spectrometric method data are not enough to remove and minimize, or even reduce considerably, the spectrum's originated noise. Two statistic methods that act locally in collected data, in the spectrum domain, have been suggested by literature to remove such remaining noises, the Noise-Adjusted Singular Value Decomposition - NASVD and Maximum Noise Fraction - MNF. These methods produce a significantly noise reduction. In this work both methods were applied in an area comprehended by two blocks, I and II, of the airborne survey that covers the west area of Mineral Province of Tapajós between Pará and Amazon states. The filtered and non-filtered data with the NASVD and MNF techniques were processed with the Lasa's parameters. The comparison of results between maps and profiles shows that both methods are valuable, since there was resolution gain in these products.
\end{abstract}

Keywords: gamma spectrometry, NASVD, MNF, noise reduction.

RESUMO. A desintegração radioativa é um processo aleatório e a estimativa de todas as medidas associadas é governada por leis estatísticas. Os perfis de taxas de contagem são sempre "ruidosos" quando utilizados períodos curtos como um segundo para cada medida. Os filtros utilizados e posteriormente as correções feitas no processamento atual de dados gamaespectrométricos não são suficientes para remover ou diminuir, consideravelmente, o ruído oriundo do espectro. Dois métodos estatísticos que atuam diretamente nos dados coletados, isto é, nos espectros, vêm sendo sugeridos na literatura para remover e minimizar estes ruídos remanescentes o Noise-Adjusted Singular Value Decomposition - NASVD e Maximum Noise Fraction - MNF. Estes métodos produzem uma redução no ruído de forma significativa. Neste trabalho eles foram implementados dentro do ambiente de processamento do software Oasis Montaj e aplicados na área compreendida pelos blocos I e II do levantamento aerogeofísico que recobre a porção oeste da Província Mineral do Tapajós, entre os Estados do Pará e Amazonas. Os dados filtrados e não-filtrados com as técnicas de NASVD e MNF foram processados com os parâmetros e constantes fornecidos pela empresa Lasa Engenharia e Prospecções S.A., sendo estes comparados. Os resultados da comparação entre perfis e mapas apresentaram-se de forma promissora, pois houve um ganho na resolução dos produtos.

Palavras-chave: gamaespectrometria, NASVD, MNF, redução de ruído.

\footnotetext{
${ }_{1}^{1}$ Departamento de Geologia Sedimentar e Ambiental, DGSA, Instituto de Geociências, IG, Universidade de São Paulo, Rua do Lago, 562, Cidade Universitária, 05508-900 São Paulo, SP, Brasil. Tel.: (11) 3091-4203 - E-mail: assisfc@yahoo.com.br

2 Departamento de Geologia e Recursos Naturais, DGRN, Instituto de Geociências, IG, Universidade Estadual de Campinas, Cidade Universitária Zeferino Vaz, Caixa Postal 6152, 13081-970 Campinas, SP, Brasil. Tel.: (19) 3521-5155 - E-mails: portugal@ige.unicamp.br; ambizuti@ige.unicamp.br

${ }^{3}$ GRM, Instituto de Geociências, Universidade de Brasília, Campus Universitário Darcy Ribeiro S/N, Asa Norte, 70910-900 Brasília, DF, Brasil. Tel.: (61) 3340-4759 Ramal: 25 -E-mail: adalene@unb.br

${ }^{4}$ AGPLA, AeroGeoPhysica Latinoamerica, Rua Albuquerque Lins, 537, g andar, Santa Cecília, 01230-001 São Paulo, SP, Brasil. Tel.: (11) 2244-2504 - E-mail: assis@agp-la.org
} 


\section{INTRODUÇÃo}

0 método gamaespectrométrico é usado principalmente para mapeamento geológico e exploração mineral, pois, as mudanças na concentração de K, U e Th acompanham as principais mudanças da litologia.

Espectrômetros modernos podem registrar no mínimo 256 canais de dados num intervalo de energia de 0-3 MeV (Fig. 1). Estes dados são somados e três janelas características de energia mostram-se interessantes ao trabalho: Potássio, Urânio e Tório. Estas janelas sofrem correções livetime e background (IAEA, 2003), remoção de radiação cósmica e radônio da atmosfera. As janelas são então submetidas ao processo de stripping (IAEA, 2003) e conversão de razões de contagem para concentrações aparentes de radioelementos (IAEA, 2003, Oasis Montaj).

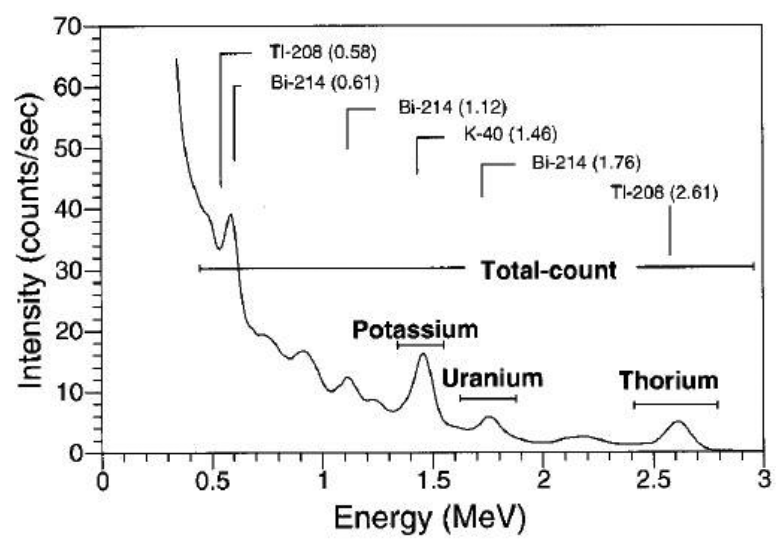

Figura 1 - Espectro típico registrado a 100 metros de altitude, onde são mostrados os picos mais proeminentes e a posição das janelas espectrais $\mathrm{K}$, U e Th (Minty, 1997).

0 processamento atual de dados gamaespectrométricos mostra que mesmo após esta etapa, ainda verifica-se ruído no espectro. Vários métodos vêm sendo sugeridos na literatura para remover este ruído remanescente, entre os quais estão o NoiseAdjusted Singular Value Decomposition - NASVD (Hovgaard, 1997a; Hovgaard \& Grasty, 1997; Minty \& McFadden, 1998) e Maximum Noise Fraction - MNF (Green et al., 1988; Dickson \& Taylor, 1998, 1999, 2000).

Estes métodos foram analisados e implementados como bibliotecas (Matlab e $\mathrm{C}_{++}$) e acoplados ao ambiente do software Oasis Montaj - Versão 5.18 da Geosoft, depois foram aplicados a uma área de estudo que compreende dois blocos, I e II do levantamento aerogeofísico que recobre a porção oeste da Província Mineral do Tapajós - sudoeste do Estado do Pará e sudeste do Estado do Amazonas e compreende uma área de $74.979 \mathrm{~km}^{2}$.

\section{ANÁLISE DE PRINCIPAIS COMPONENTES - APC}

A análise dos componentes principais (do inglês Principal Component Analysis - PCA) é um método para decompor uma matriz de dados $m$ de posto $r$, como uma soma de matrizes de posto igual a 1, onde posto é um número que expressa a dimensão de uma matriz (Noble \& James, 1977).

Dado um conjunto de $m$ vetores de dados (organizados na forma de uma matriz), a idéia básica consiste em achar uma transformação linear que cumpra uma exigência. A premissa mais importante do método Análise de Principais Componentes (APC) é que se considera que o sinal, ou informação contida no dado, é aquela que tem maior variância, ao contrário do ruído que possui (por hipótese) menor variância.

Pode-se mostrar que essa transformação é uma rotação (Noble \& James, 1977) de modo que se preserva a distância entre os dados. Além disso, este novo conjunto de vetores transformados fica indexado na ordem decrescente de variância. Isto mostra quais dados têm maior influência sobre 0 conjunto, segundo 0 critério de maior variância.

Sua aplicação mais óbvia é a compressão de dados, isto é, tendo um conjunto grande de dados, pode-se descobrir, após a transformação, as direções mais importantes, projetar os dados originais nestas direções encontradas e aplicar a transformação inversa para as direções originais. Desta forma, reduz-se consideravelmente 0 número de variáveis, havendo perdas de informações, mas de forma minimizada, ficando as informações (sinal) concentradas, fato que simplifica a análise deste conjunto de dados.

Desta forma, considere um conjunto " $A$ " de $m$ espectros (Fig. 2) com 256 canais (poderia ser 512 ou 1024 canais, dependendo da resolução do equipamento), as principais componentes deste conjunto $A_{(m \times 256)}$ de $m$ espectros são os autovaIores da matriz de covariância cov(A). Este tipo de análise produz 256 autovetores, os quais correspondem às Principais Componentes, e 256 autovalores que são as medidas da contribuição de cada autovetor em relação ao conjunto de entrada $A$. Eles são as variâncias de cada autovetor, e somados resultam na variação total no espectro.

Um espectro observado pode ser reconstruído pelos autovetores, multiplicando cada autovetor com uma apropriada amplitude e somando estes produtos. Em notação matricial:

$$
A=C V
$$

onde $A_{(m \times 256)}$ é 0 espectro de entrada, $C_{(m \times 256)}$ é a concentração (amplitude) e $V_{(256 \times 256)}$ é a matriz com os componentes principais (autovetores). 


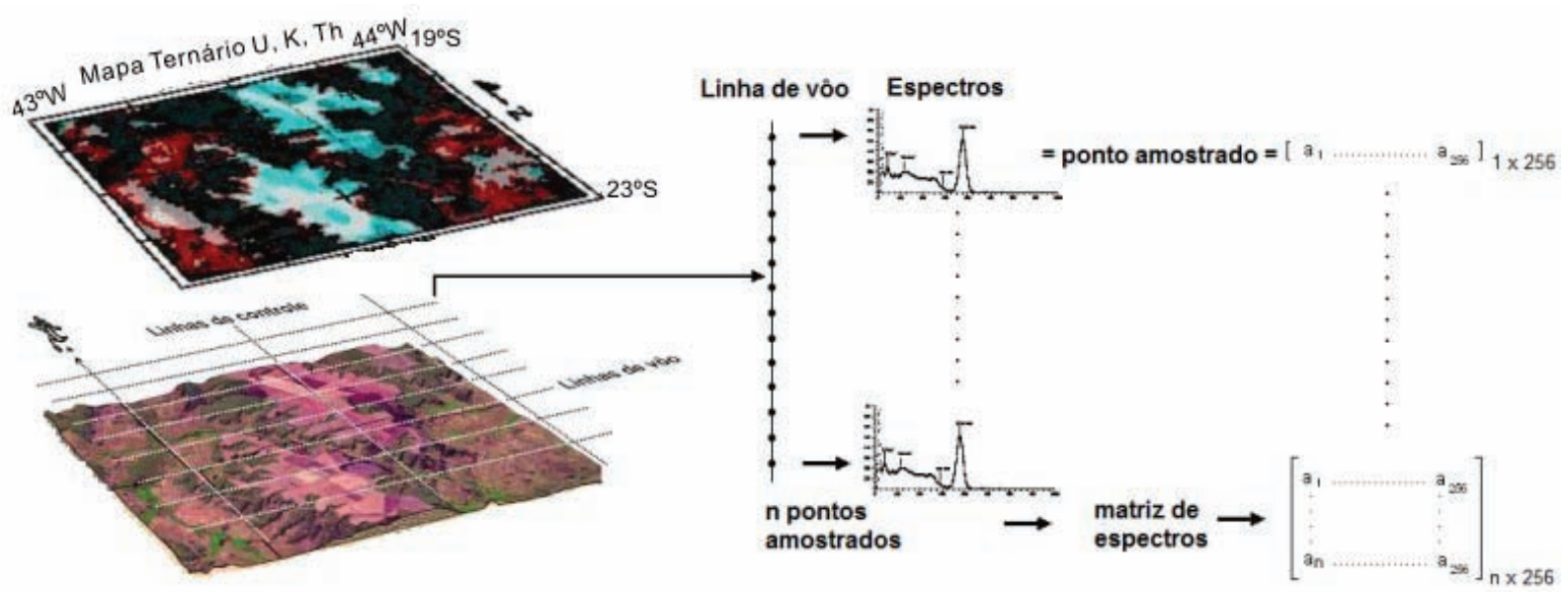

Figura 2 - Diagrama ilustrativo dos pontos amostrados em uma linha de vôo e sua respectiva matriz de espectros (" $M_{\text {original }}$ ").

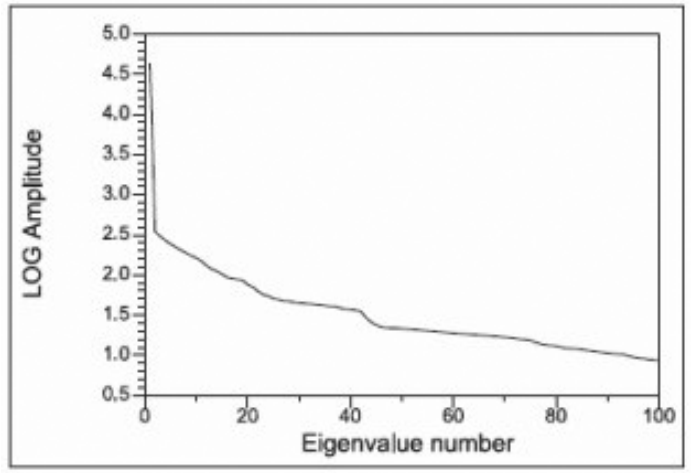

Figura 3 - Curva que representa os autovalores de uma APC sem nenhum tipo de normalização (Minty, 2000).

Assim, a APC pode ser usada para remover o ruído de espectros multicanais se 0 sinal for fortemente correlacionado dentro do espectro de entrada e o ruído não. Isto é possível porque o sinal concentra-se, em sua maior parte, nas componentes de baixa ordem. 0 ruído por sua vez não tem uma boa correlação entre os canais razão de contagem, tendendo a espalhar-se por todas as componentes. Então, pode-se remover uma grande parte do ruído reconstruindo o espectro "suavizado" com algumas componentes de baixa ordem.

Para exemplificar, na Figura 3, estão representados os autovalores em relação às amplitudes de uma APC aplicada a um vôo teste. Observam-se as grandes amplitudes concentradas nos primeiros autovalores e que elas tendem, de maneira lenta, para baixos valores, isto é, o sinal persiste nas componentes restantes.

Todavia, verificando um espectro típico gamaespectrométrico (Fig. 1) nota-se que os canais de baixas energias têm razão de contagem superior muitas vezes maior que os canais de altas energias. Isto significa que estes canais de baixas energias dominam completamente a APC. Não há nada de errado nisto, uma vez que os canais (variáveis) são todos expressos na mesma unidade (counts/s). 0 problema é que os canais de baixas energias possuem erros com alta variância em relação aos canais de altas energias. Isto significa que o ruído, em alguns canais, terá maior peso na APC que o sinal em outros. Uma saída para resolver este problema é normalizar o ruído em todos os canais, igualando os pesos do mesmo no espectro.

Numa primeira aproximação, pode-se assumir que o erro da variância do canal razão de contagem é a média da razão de contagem para este canal dentro do espectro de entrada (bruto). Assim, se para um determinado canal a média da razão de contagem é $n$, então, pode-se normalizar este canal em cada espectro pela divisão do canal da razão de contagem por $\sqrt{n}^{1}$.

\footnotetext{
${ }^{1}$ Por que dividir por $\sqrt{n}$ e não por $n$ ? Se uma variável aleatória $x$ tem uma variância de var $(x)$, então a variância da função: $y=f(x)=\frac{x}{a}$, onde $a$ é uma constante, é dada por $\operatorname{var}(y)=\frac{\operatorname{var}(x)}{a^{2}}$. Desta segue que se a função tem um erro na variância $n$, e se este for dividido por $\sqrt{n}$, então $\operatorname{var}(f)=1$ (Gilmore \& Hemingway, 1995).
} 
A Figura 4 mostra a mesma APC, do mesmo vôo teste mostrado na Figura 3, onde foi aplicada uma normalização ao espectro de entrada. Observa-se que os autovalores tendem rapidamente para um patamar próximo a zero (Fig. 4), que representa as componentes que contem essencialmente ruído. Com a normalização aplicada ao espectro de entrada e a variância do erro unitário, as amplitudes do ruído das componentes devem ser as mesmas, aproximadamente.

Neste ponto é que os métodos NASVD e MNF atuam, pois, ambos fazem essencialmente uma APC dos espectros brutos. A principal diferença entre eles é como eles normalizam os espectros brutos em relação ao ruído. Os dois métodos serão apresentados em seguida.

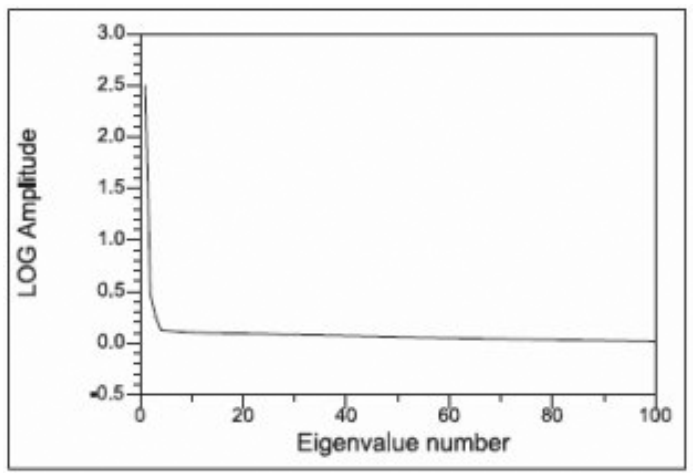

Figura 4 - Curva que representa os autovalores de uma APC depois de normalizado (Minty, 2000).

\section{OS MÉTODOS NASVD E MNF}

NASVD (Noise-Adjusted Singular Value Decomposition) é uma técnica elaborada para remover ruído de dados gamaespectrométricos brutos usando a análise de componentes espectrais. As componentes de ordem inferior são interpretadas como sinal, e as de ordem superior como ruído, sendo estas últimas desprezadas. A partir deste ponto, reconstrói-se o espectro original somente com os componentes de ordem inferior. 0 procedimento NASVD baseia-se na análise estatística multivariada para extrair 0 espectro dominante dos dados brutos. 0 caminho mais eficaz para isto é usar componentes ortogonais, pois simplifica os cálculos matriciais (Noble \& James, 1977).

Há dois aspectos associados ao uso da APC para espectros multicanais que devem ser observados:

1) a variância associada a cada canal deve ser a mesma, isto é, normalização do espectro de entrada para variância unitária em cada canal. Hovgaard (1997a, 1997b) sugeriu uma solução simples: como a variância de um canal razão de contagem é a mesma, assim como a razão de contagem média para este mesmo canal, o melhor ajuste do espectro médio para cada espectro de entrada dá uma boa estimativa da razão de contagem média (e, portanto, da variância) para cada canal. Assim, o espectro de entrada é escalonado para uma variância unitária em cada canal pela divisão destes pela raiz quadrada do melhor ajuste do espectro médio para cada um dos espectros de entrada. Isto é,

\section{Ajuste do ruído (NA - Noise adjusted)}

Sum(i) $(=$ a contagem Total $)=\sum_{j=1}^{m} A(i, j)$, onde:

$n=$ total de espectros em uma linha de vôo,

$j=1, \ldots, j$-ésimo canal,

$i=$ i-ésimo espectro ;

\section{Espectro normalizado:}

$S(i, k)=\frac{A(i, k)}{\operatorname{Sum}(i)}$, onde $k=1 \ldots$ késimo canais;

$S N(k)=\sum_{k=1}^{n} S(i, k)$

$S N(i)=\sum_{i=1}^{n} S(i, k)=N^{\circ}$ de pontos amostrados $=N^{\circ}$ de linhas da matriz de entrada;

\section{Espectro representativo normalizado:}

$$
S T_{k}(k)=\frac{S N(k)}{\sum_{i=1}^{n} S N(i)}
$$

\section{Espectro com ruído ajustado:}

$$
A_{\text {new }}(i, j)=A(i, j) * \frac{1}{\sqrt{S T_{k}(j) * \operatorname{Sum}(i)}}
$$

onde:

$$
\text { Filtro_NA }=\frac{1}{\sqrt{S T_{k}(j) * \operatorname{Sum}(i)}}
$$

2) A APC requer que os dados estejam centrados na média para que se obtenham melhores resultados. Para tanto é necessário aplicar a decomposição SVD (Noble \& James, 1977) que analisa a dispersão dos dados de forma mais eficaz que a média simples.

0 espectro com ruído ajustado, $A_{\text {new }}$, é o elemento de entrada do algoritmo SVD. 
Qualquer matriz $\mathrm{A}_{(m \times n)}$, onde $m \times n$, pode ser reescrita como produto de três matrizes domínio (Noble \& James, 1977):

$$
A=U \Sigma V^{T}
$$

onde: $U\left(m_{\text {espectros }} \times n_{\text {canais }}\right)$ é uma matriz de coeficientes, $\Sigma(n \times n)$ é matriz diagonal de valores singulares, os autovalores, $\left(\sigma_{1} \geq \sigma_{2} \geq \ldots \geq \sigma_{n}>0\right), V^{T}(n \times n)$ é a matriz dos autovetores, que são as componentes espectrais dos dados.

Os autovetores de $A^{T} A$ são as colunas de $V$ (ortogonal) e os autovalores são 0 quadrado dos elementos da diagonal de $\Sigma$. A equação (4) é usada para calcular os autovetores $(V)$ e amplitudes $(U \Sigma)$ de $A$. Os autovetores são re-escalonados multiplicando pela soma das contagens em cada espectro de entrada. As primeiras componentes de $\Sigma$ e $V$ contêm a maior parte do sinal. Portanto, o ruído é removido reconstruindo o espectro com autovetores e amplitudes de baixas ordens.

MNF - A transformação MNF (Maximum Noise Fraction) é um procedimento estatístico no âmbito da APC e representa um aprimoramento desta. Uma das grandes limitações da APC para a remoção do ruído é que nem sempre se observa, nas componentes principais geradas, um aumento da razão sinal/ruído de forma crescente em relação aos autovalores (Green et al., 1988). Essa tendência aumenta com a complexidade dos dados. A vulnerabilidade da APC para a eliminação do ruído é resultante da priorização da variância no ordenamento dos dados, ao invés da razão sinal/ruído. Assim sendo, Green et al. (1988) propuseram 0 procedimento estatístico MNF, que enfoca o ruído em depreciação da variância do dado. Este é derivado e análogo à transformação APC, distinguindo-se apenas quanto ao critério para a geração das componentes. 0 MNF promove a maximização do ruído ao invés da variância dos dados, o que proporciona um ordenamento que reflete a qualidade do sinal. Ao invés de escolher uma nova componente para maximizar a variância, assim como a transformação da componente principal faz, agora se escolhe maximizar a razão sinal/ruído (Green et al., 1988).

Considerando o conjunto de dados multivariados composto por $p$ canais, $Z_{i}(x), i=1, \ldots, n$, onde $x$ representa 0 espectro, assume-se que

$$
Z(x)=S(x)+N(x),
$$

onde $S(x)$ e $N(x)$ são os componentes: sinal e ruído não correlacionados de $Z(x)$. Então:

$$
\operatorname{Cov}\{Z(x)\}=\Sigma=\Sigma_{S}+\Sigma_{N},
$$

onde $\Sigma_{S}$ e $\Sigma_{N}$ são as matrizes de covariância de $S(x)$ e $N(x)$.
A fração do ruído do i-ésimo canal é igual à razão entre a variância do ruído e a variância total do canal, isto é $\operatorname{Var}\left\{N_{i}(x)\right\} / \operatorname{Var}\left\{Z_{i}(x)\right\}$. Desta forma, estabelece-se uma matriz de covariância do índice de fracionamento do ruído nos mesmos moldes de uma matriz de covariância em APC.

De caráter linear, a transformação MNF é expressa como:

$$
Y i(x)=a_{i}{ }^{T}(x), i=1 \ldots, n ;
$$

usando argumento similar a APC, temos que $a_{i}$ representa os autovetores de $\Sigma^{-1} \Sigma_{N}$ e $\lambda_{i}$ aos autovalores associados, equivalendo à fração do ruído em $Y i(x)$. Portanto, a partir dessa definição verifica-se, para a transformação MNF, um ordenamento dos autovalores de acordo com a qualidade do sinal, onde:

$$
Y i(x) \cdot \lambda_{1} \geq \lambda_{2} \geq \ldots \geq \lambda_{p} .
$$

De acordo com esta formulação matemática, a grande diferença do MNF para a APC é a substituição da matriz de covariância-variância dos dados pela matriz relativa aos índices de fracionamento de ruído. A utilização de uma ou outra matriz proporciona a maximização de um determinado tipo de informação.

\section{RESULTADOS}

Os filtros MNF e NASVD foram implementados e testados em uma linha de vôo teste. Os espectros brutos e filtrados resultantes foram comparados (Figs. 5 e 6) com a finalidade de observar a atuação dos filtros, onde pode-se observar a suavização resultante das filtragens com oito componentes. A forma do perfil filtrado varia com a quantidade de componentes, isto é, quanto maior o número de componentes utilizadas mais próxima será a forma do perfil filtrado em relação ao espectro bruto e também maior será a quantidade de ruído recuperado.

\section{APLICAÇÃO DOS FILTROS A UMA ÁREA REAL}

A área de estudo localiza-se na Amazônia, abrangendo parte do sudoeste do Estado do Pará e sudeste do Estado do Amazonas, compreendendo cerca de $74.979 \mathrm{~km}^{2}$ (Fig. 7) limitada pelos paralelos $05^{\circ} 00^{\prime}$ e $08^{\circ} 00^{\prime}$ de latitude sul e pelos meridianos $55^{\circ} 30^{\prime}$ e $58^{\circ} 30^{\prime}$ de longitude oeste de Greenwich.

\section{DADOS GAMAESPECTROMÉTRICOS}

0 aerolevantamento foi efetuado a altura constante de $100 \mathrm{~m}$ e com linhas de vôo e controle espaçados entre 1.000 e 13.000m, respectivamente, tendo as linhas de vôo à direção N-S, e linhas de controle E-W. Ao longo dos perfis foram realizadas leituras 


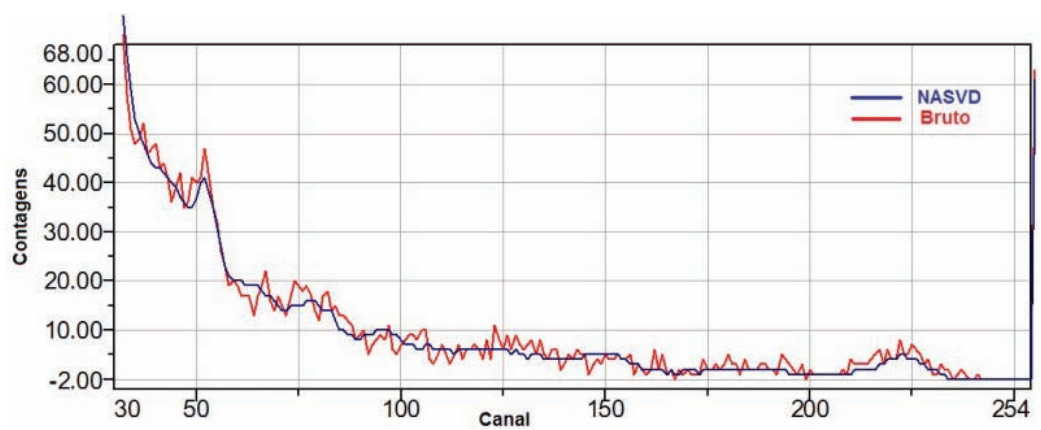

Figura 5 - Comparação entre um espectro original de Urânio (linha vermelha) e o mesmo filtrado pelo NASVD (linha preta) em um ponto amostrado, utilizando 8 componentes.

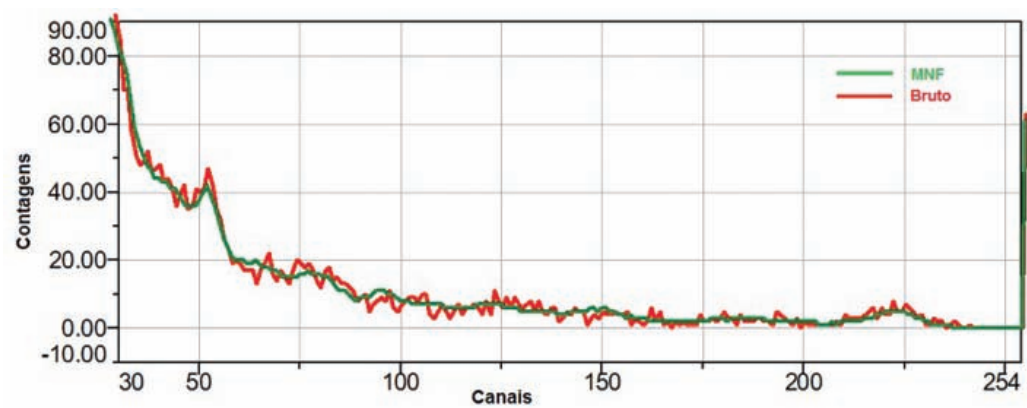

Figura 6 - Comparação entre um espectro original de Urânio (linha preta) e o mesmo filtrado pelo MNF (linha verde) em um ponto amostrado, utilizando 8 componentes.

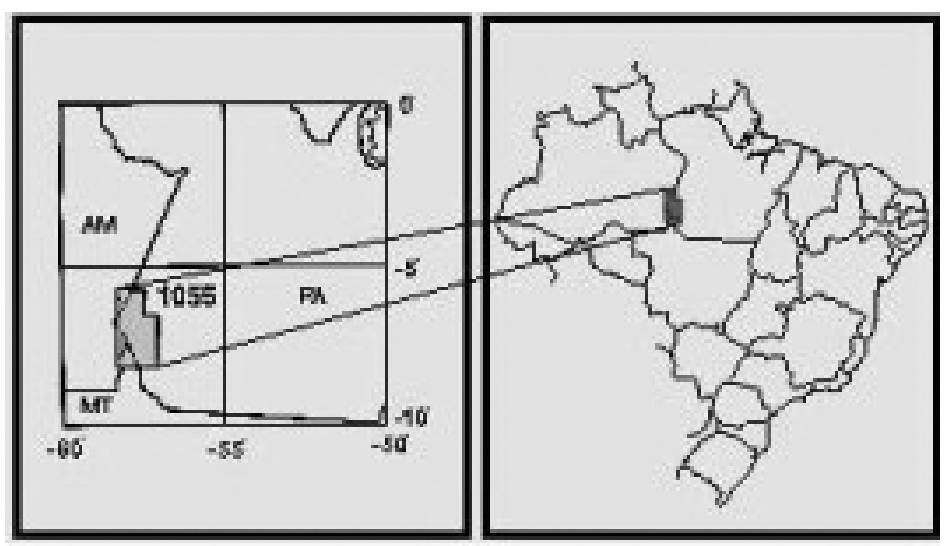

Figura 7 - Localização do levantamento aerogeofísico do Projeto Província Aurífera do Tapajós (CPRM, 2008).

do gamaespectrômetro por segundo, posicionadas com sistema de observação de satélite GPS, de precisão melhor do que 10m. Os dados foram registrados a bordo da aeronave, digital e analogicamente, pelo sistema de aquisição GEODAS.

Em seguida eles foram processados pela empresa Lasa, e reprocessados neste trabalho utilizando-se os coeficientes e constantes do Relatório Final do Levantamento dos Dados Magnetométricos e Gamaespectrométricos (Lasa-Fugro, 1998).
Na primeira etapa efetuou-se uma análise estatística dos espectros filtrados, numa tentativa de estabelecer um procedimento de escolha da quantidade de componentes, relacionada com um determinado filtro, para que se possa obter uma filtragem com melhor qualidade. Este procedimento consiste na diferença entre o espectro bruto e espectro filtrado que representa teoricamente o ruído, mas na prática sempre há um mínimo de sinal residual (Fig. 8). Fazendo à média desta diferença, esta tenderá a zero. 


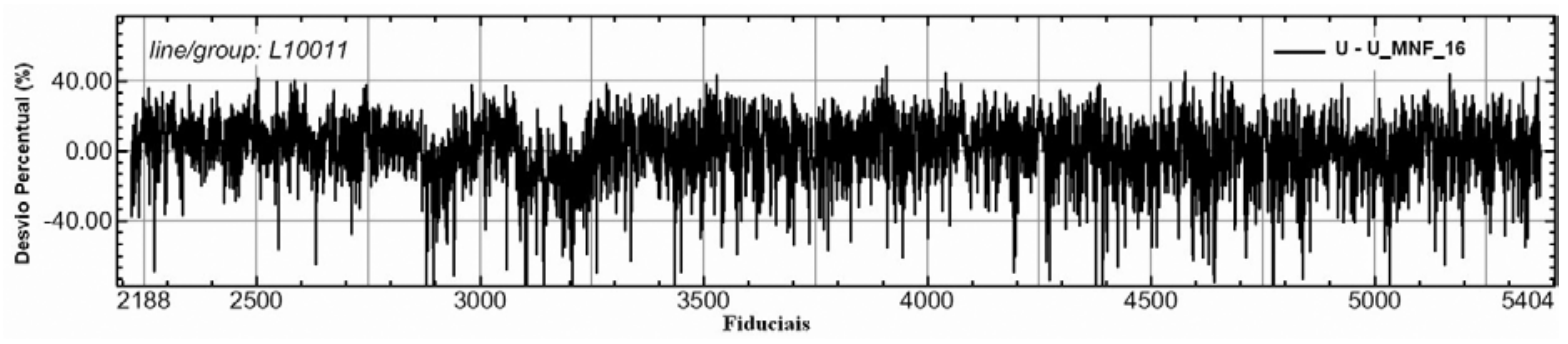

Figura 8 - Representação visual da diferença entre $U_{\text {bruto }}$ e $U_{\text {filtrado }}$ com MNF - 16 componentes. 0 ruído varia dentro de um intervalo, cuja média está próxima a zero. Neste caso há sinal, com valor inexpressivo (Tab. 1).

Esta tendência pode então servir como parâmetro para indicar qual filtro, com determinada quantidade de componentes, pode ser adotado como melhor escolha.

A diferença entre 0 espectro bruto e o espectro filtrado fica melhor representada de forma percentual. Assim, esta diferença pode ser da forma:

$$
\text { Diferença percentual }=100 \times \frac{\text { janela filtrada }- \text { janela bruto }}{\text { janela bruto }}
$$

A expressão matemática apresentada acima foi aplicada para as janelas de Urânio, Potássio e Tório extraídos dos espectros brutos e filtrados para todo o banco de dados da área de estudo.

A Tabela 1 mostra 0 desvio percentual do Urânio, Potássio e Tório em relação ao filtro utilizado com seu respectivo desvio padrão. Analisando os dados desta tabela observa-se que o filtro MNF possui os menores desvios percentuais em relação ao filtro NASVD, cujos valores de desvios percentuais são os maiores e o filtro Passa-Baixa, aqui representado com finalidade apenas comparativa.

Tabela 1 - Desvio percentual das janelas de interesse em relação aos filtros NASVD, MNF e Passa-Baixa, variando-se a quantidade de componentes.

\begin{tabular}{|c|c|c|c|c|c|c|}
\cline { 2 - 7 } \multicolumn{1}{c|}{} & Urânio & $\sigma \mathrm{U}$ & Potássio & $\sigma \mathrm{K}$ & Tório & $\sigma \mathrm{Th}$ \\
\hline NASVD_4 & 24,64 & 18,28 & 13,54 & 18,68 & 30,33 & 30,22 \\
\hline NASVD_8 & 23,93 & 17,58 & 13,34 & 15,76 & 32,10 & 30,24 \\
\hline NASVD_16 & 23,96 & 17,30 & 13,56 & 15,60 & 33,03 & 28,50 \\
\hline NASVD_32 & 24,11 & 16,11 & 14,67 & 13,05 & 34,25 & 25,00 \\
\hline NASVD_64 & 24,15 & 15,34 & 16,02 & 8,65 & 36,03 & 23,10 \\
\hline MNF_4 & 1,48 & 20,01 & 1,10 & 18,03 & 8,31 & 39,64 \\
\hline MNF_8 & 0,75 & 20,08 & 0,78 & 18,04 & 5,98 & 37,89 \\
\hline MNF_16 & 0,68 & 20,06 & 0,99 & 17,76 & 4,59 & 37,89 \\
\hline MNF_32 & 0,70 & 19,18 & 2,10 & 14,48 & 2,24 & 28,30 \\
\hline MNF_64 & 1,50 & 15.71 & 3.46 & 8.40 & 0.58 & 22.71 \\
\hline LOW PASS & 1,91 & 13,96 & 78,14 & 28,14 & 56,37 & 30,96 \\
\hline
\end{tabular}

0 fato de 0 filtro NASVD possuir altos valores no desvio percentual indica que este método não separa o sinal e ruído de forma eficiente em relação ao método MNF, isto é, estes valores indicam que ainda há uma quantidade significativa de sinal residual (Figs. 13 e 14). Mas, este fato não o invalida como filtro, apenas mostra que para este conjunto de dados ele não foi tão eficiente quanto 0 filtro MNF.

A escolha do filtro e da quantidade de componentes pode ser feita considerando os menores valores de desvios percentuais; porém, isto vai depender muito da experiência do geofísico que está processando os dados gamaespectrométricos.

Numa primeira escolha, os filtros NASVD e MNF com 8 e 16 componentes respectivamente, seriam uma escolha, pois no caso MNF o valor apresentado de desvio percentual do Urânio é o menor e, sendo o canal mais ruidoso, 0 torna o mais importante numa possível escolha. Apesar do desvio percentual do Potássio não ser o menor, seu valor de desvio padrão é menor em relação ao valor do filtro MNF com 8 componentes. Quanto ao Tório é 0 menos afetado pelo ruído e como no processamento usa-se diretamente os dados brutos sem filtragem, este não foi analisado em detalhes. Em relação ao filtro NASVD a escolha é um pouco mais difícil, pois o valor de desvio percentual é muito alto em relação aos valores do filtro MNF, indicando perda significativa de sinal. Assim, a escolha do filtro NASVD com 8 componentes seria a mais indicada por possuir os menores valores de desvio percentual para o Urânio e Potássio. 0 desvio percentual foi aplicado também ao filtro Passa-Baixa (Low-Pass). Apesar de 0 desvio percentual ser pequeno para 0 Urânio, fazendo uma inspeção visual do canal filtrado em relação ao bruto (Fig. 9) observa-se uma forte suavização, ocasionando uma significativa perda de sinal, além disto, os valores de desvio percentual para o Potássio e Tório serem muitos altos.

0 cálculo da diferença entre os dados brutos e filtrados fornece uma forma interessante de estimar a qualidade da filtragem, mas uma inspeção visual dos dados filtrados em relação aos dados brutos também é importante, pois dá a noção da força de suavização do filtro utilizado. Isto pode ser feito perfilando 0 canal bruto e o filtrado de interesse (por exemplo: Urânio) e comparando-os.

A segunda etapa foi analisar, visualmente, o efeito do filtro Passa-Baixa (freqüência de corte $=3$ fiduciais) em dados bru- 


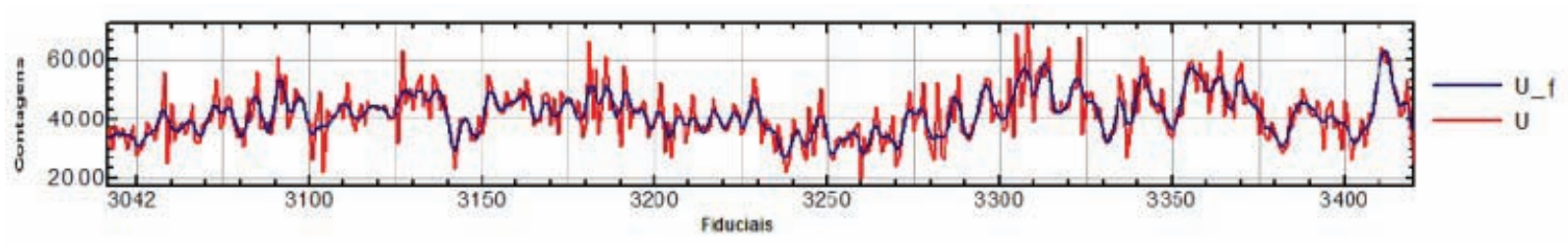

Figura 9 - Comparação entre U bruto e o mesmo filtrado pelo Passa-Baixa (freqüência de corte = 3 fiduciais).

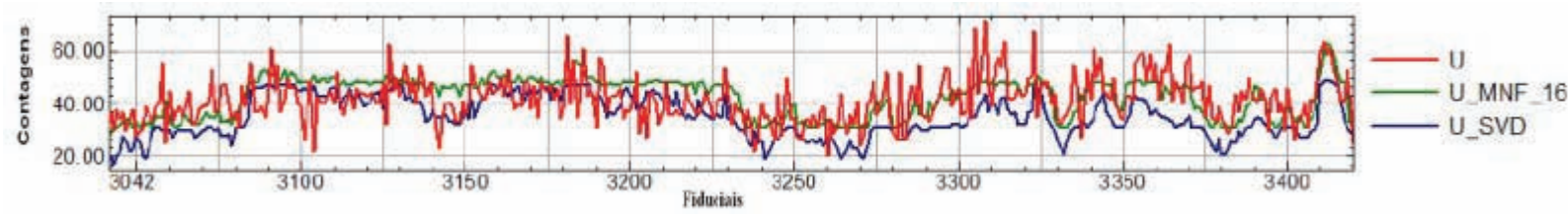

Figura 10 - Comparação entre U bruto e o mesmo filtrado pelo MNF (16 componentes) e NASVD (8 componentes).

tos de Urânio, Potássio e Tório e comparando-os com seus dados brutos. Observando os perfis pode-se ver que a suavização é muito forte, havendo perda de informações de forma expressiva, exemplificado na Figura 9, onde o canal bruto (vermelho) do U é comparado com o canal filtrado (azul). Da mesma forma pode-se analisar o produto das filtragens NASVD e MNF no canal Urânio e compará-los aos respectivos canais brutos. A Figura 10 mostra 0 canal bruto (vermelho) do Urânio, os respectivos canais filtrados pelo NASVD (azul) e MNF (verde). Neste caso, pode-se observar que a suavização está ajustada em relação aos dados brutos, isto é, a subtração de ruído não foi excessiva, preservando mais o sinal contido nos dados.

A etapa final concentrou-se na confecção de mapas, basicamente do Urânio e Potássio, dos dados gamaespectrométricos filtrados ou não e pós-processados. Do total de mapas confeccionados alguns foram selecionados e agrupados de forma a facilitar a análise visual dos resultados.

Seguindo a escolha feita através da Tabela 1 os mapas de Urânio correspondentes aos filtros NASVD e MNF com 8 e 16 componentes respectivamente, foram colocados lado a lado e comparados aos mapas de Urânio bruto e filtrado com PassaBaixa com 3 fiduciais. A Figura 11 mostra os mapas de Urânio pós-processados sem filtragem (a), NASVD (b), Passa-Baixa (c) e MNF (d). Também mostra uma área selecionada (retângulo branco) e ampliada onde foram assinaladas feições (anomalias) em círculos pretos e identificados pelas letras " $A$ ", " $B$ ", " $C$ " e " $D$ ", onde se pode observar 0 aumento do ganho na resolução; conseqüentemente, os contornos destas anomalias ficaram mais bem definidos nas Figuras 11(b) - filtro NASVD e 11(d) - filtro MNF em relação às Figuras 11(a) - sem filtragem e 11(c) Passa-Baixa.
As áreas selecionadas apenas exemplificam detalhes da perda ou ganho de resolução, dependendo do tipo de filtro aplicado. Como visto anteriormente, pode-se analisar através da Tabela 1 , o desvio percentual de cada filtro em relação ao conjunto de dados estudado, porém é interessante visualizar esta diferença em forma de mapa. Assim, a Figura 12 mostra três mapas das diferenças entre 0 Urânio bruto (sem filtragem) pós-processado com os filtros Passa-Baixa (a), MNF - 16 componentes (b), NASVD - 8 componentes (c). Estes mostram basicamente 0 ruído subtraído pelos filtros. Todavia, inspecionando-se com cuidado os mapas das Figuras 12(b) e 12(c) pode-se observar a existência de sinal em áreas localizadas, conforme os círculos pretos identificados por "A" e "B", sendo que a quantidade de sinal remanescente depende do número de componentes utilizados pelo filtro. Mas no caso do filtro Passa-Baixa, Figura 12(a), o sinal é distribuído através de todo o mapa, isto é, ele é encontrado em toda a sua extensão demonstrando de forma visual que 0 efeito deste filtro é muito forte, isto é, cortando (subtraindo) acima de um determinado valor indistintamente tanto ruído como sinal, como já visto em perfil na Figura 9. Quanto aos mapas das Figuras 12(b) e 12(c), eles mostram visualmente que a segregação entre 0 sinal e o ruído foi efetivada. Além disso, a análise destes mapas torna-se importante para estimar 0 aumento ou a diminuição da quantidade de componentes utilizadas, conforme a feição que se procura.

De forma análoga fez-se a mesma análise com os mapas do Potássio utilizando a Tabela 1 para escolher os filtros com menor desvio percentual. A Figura 13 mostra mapas de Potássio pósprocessados escolhidos: sem filtragem (a), filtragem NASVD -64 componentes (b), filtragem Passa-Baixa - 3 fiduciais (freqüência de corte) (c) e filtragem MNF - 8 componentes (d) com suas respectivas áreas em detalhamento e ampliadas. 


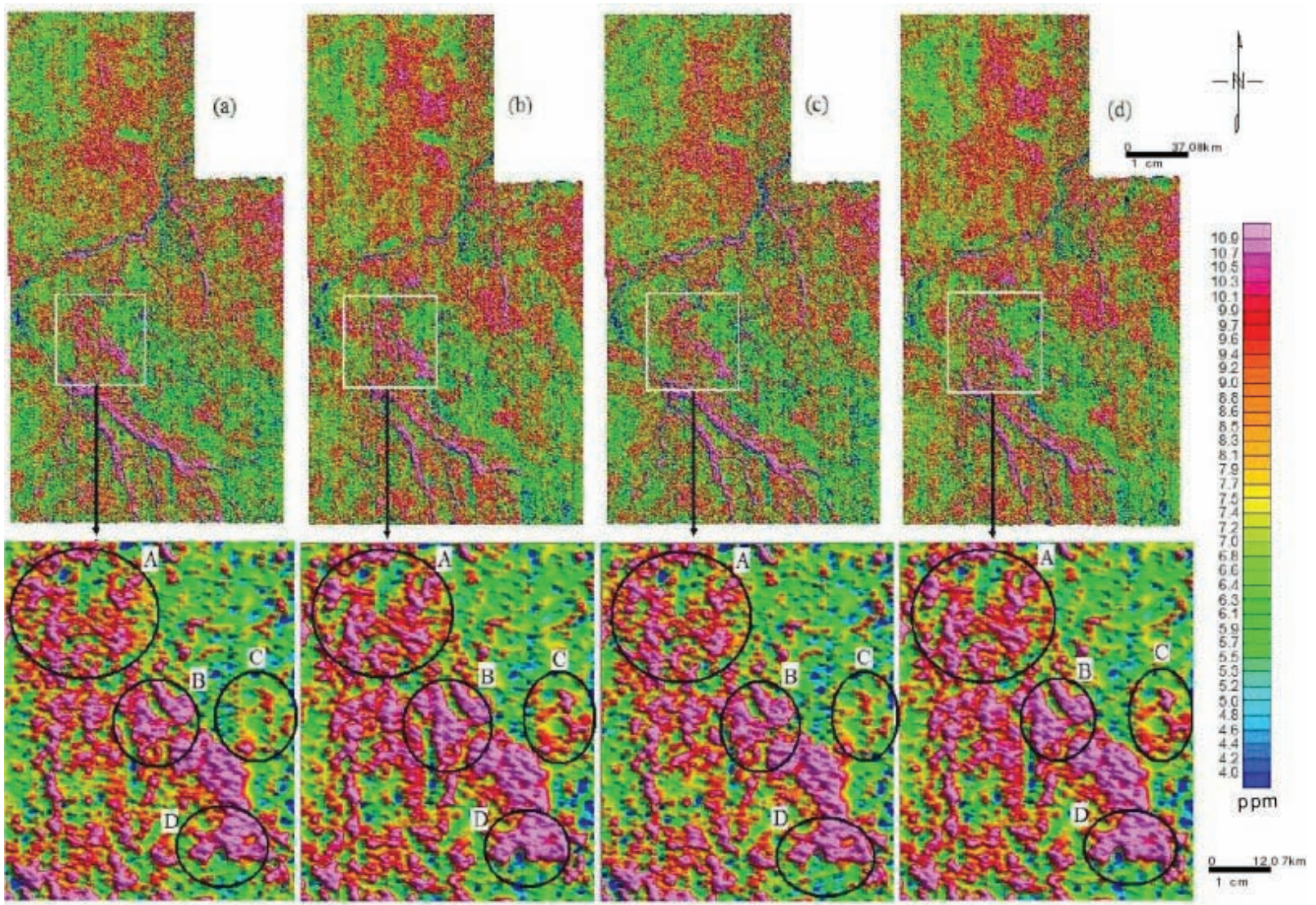

Figura 11 - Mapas de Urânio pós-processados: (a) sem filtragem, (b) filtragem NASVD com 8 componentes, (c) filtragem com Passa-Baixa (Low Pass) - 3 fiduciais (freqüência de corte) e (d) filtragem MNF com 16 componentes com suas respectivas áreas em detalhamento e ampliação.

0 Potássio tem baixa energia em relação ao Urânio e Tório, sendo mais suscetível ao ruído. Observando os valores de desvio percentual do filtro MNF e NASVD na Tabela 1 escolheu-se 0 filtro com 16 e 64 componentes, respectivamente. 0 valor de desvio percentual escolhido do MNF é o segundo menor (o menor é MNF - 8 componentes), mas este valor está bem próximo daquele e possui desvio padrão menor. Esta escolha seguiu a mesma lógica de escolha utilizada para o Urânio.

Quanto ao filtro NASVD, segue o mesmo padrão em relação ao Urânio, possuindo valores altos de desvio percentual, que significa que a interface de separação sinal/ruído não está bem definida para este conjunto de dados. Uma segunda escolha é o filtro NASVD com 64 componentes, pois tem um desvio padrão menor, apesar de possuir o maior valor de desvio percentual.

Este fato, como já dito anteriormente, não inviabiliza o filtro NASVD e o procedimento de escolha por desvio percentual apenas requer um estudo mais detalhado com várias áreas conhecidas, tanto na geologia quanto na gamaespectrometria. Todos os mapas resultantes da filtragem com NASVD com 4, 8, 16, 32 e 64 componentes foram analisados chegando-se à conclusão de que o filtro com 64 componentes realmente é 0 que possui a melhor resolução.

Analisando a Figura 13 podem-se perceber as diferenças na resolução das feições (anomalias) com maior detalhamento nas imagens ampliadas de pequenas áreas. As imagens ampliadas que foram filtradas com 0 MNF e NASVD (detalhamento mostrado nos círculos pretos denominados "A" e "B") destacam-se, pois apresentaram o maior ganho em resolução nas imagens correspondentes aos não filtrado e filtrado pelo Passa-Baixa. Repetindo o procedimento como efetuado com os mapas de Urânio, fizeram-se os mapas de diferenças entre os produtos filtrados com 0 mapa de Potássio bruto pós-processado. A Figura 14 mostra estes mapas. Os mapas da Figuras 14(b) e 14(c) mostram basicamente ruídos, tendo valores muito próximos a zero e que a quantidade de sinal remanescente é muito pequena. Porém, a Figura 14(a) mostra uma visualização muito parecida com a do 

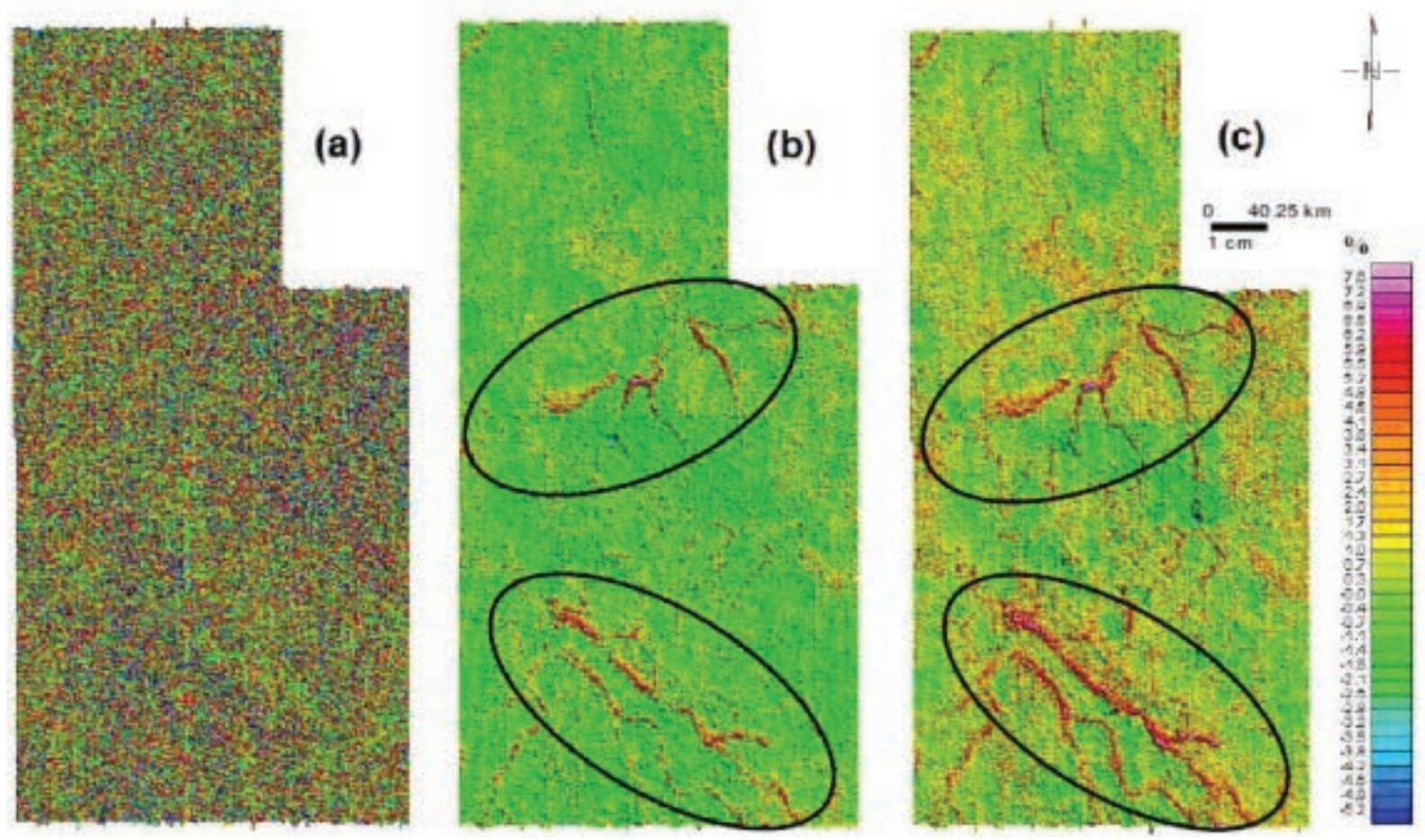

Figura 12 - Mapas das diferenças entre Urânio bruto pós-processado com os filtros Passa-Baixa - 3 fiduciais (a), MNF - 16 componentes (b), NASVD - 8 componentes (c).

Urânio. A quantidade de sinal é mais expressiva que a dos outros filtros e distribuído por toda a área estudada, demonstrando que 0 seu efeito foi muito forte, isto é, o sinal é excessivamente cortado.

Todos os mapas dos filtros escolhidos ainda terão que passar por nivelamento (efeitos visuais aparentes no sentido norte-sul), mas esse processo será muito facilitado com a atuação dos filtros MNF e NASVD, porque tais filtros minimizam estes efeitos.

\section{CONCLUSÃO}

Os filtros MNF e NASVD atuam utilizando a estatística como alicerce, mostraram ser alternativas bastante promissoras como ferramentas de auxílio no processamento de dados gamaespectrométricos.

0 destaque é que 0 filtro MNF conseguiu ter uma atuação muito mais vantajosa que os filtros habituais que trabalham com freqüência de corte do que o filtro NASVD. 0 resultado apresentado por este filtro mostra, através dos perfis e mapas, que com certeza os produtos finais terão uma sensível melhora em resolução e, conseqüentemente, melhor qualidade.

Quanto ao filtro NASVD, apesar de apresentar um resultado bom, ainda precisa ser mais bem estudado, aplicando-0 em várias áreas com cobertura conhecida em aerolevantamento e geologia, pois, apesar de se conseguir minimizar o ruído e melhorar a qualidade dos mapas, o resultado ficou abaixo do esperado, necessitando, portanto, de melhor investigação. Mas este fato não 0 inviabiliza, apenas indica que ele deve ser melhor estudado, talvez alterando a forma de aplicação, como sugerido em algumas publicações que fazem uso de agrupamentos (por exemplo: Minty \& McFadden, 1998).

Outro aspecto é 0 da escolha do filtro a ser utilizado. Apesar deste trabalho sugerir um procedimento de escolha da quantidade de componentes através de diferenças entre os dados brutos e dados filtrados, é importante deixar bem claro que decisões a serem tomadas dependem, e muito, da experiência do geofísico que está processando, ampliando o leque de opções que ele possa utilizar.

Portanto, os resultados descritos neste trabalho demonstram que os filtros NASVD e MNF são alternativas que contribuirão, de forma positiva, para a melhoria da qualidade dos produtos finais.

\section{AGRADECIMENTOS}

Os autores agradecem a empresa FUGRO, mais especificamente a Luiz Braga e César Marinho por ceder gentilmente os dados da área de estudo. Também aos amigos geofísicos Luciano Konzen e Leandro Moutinho pelas diversas discussões e sugestões. 

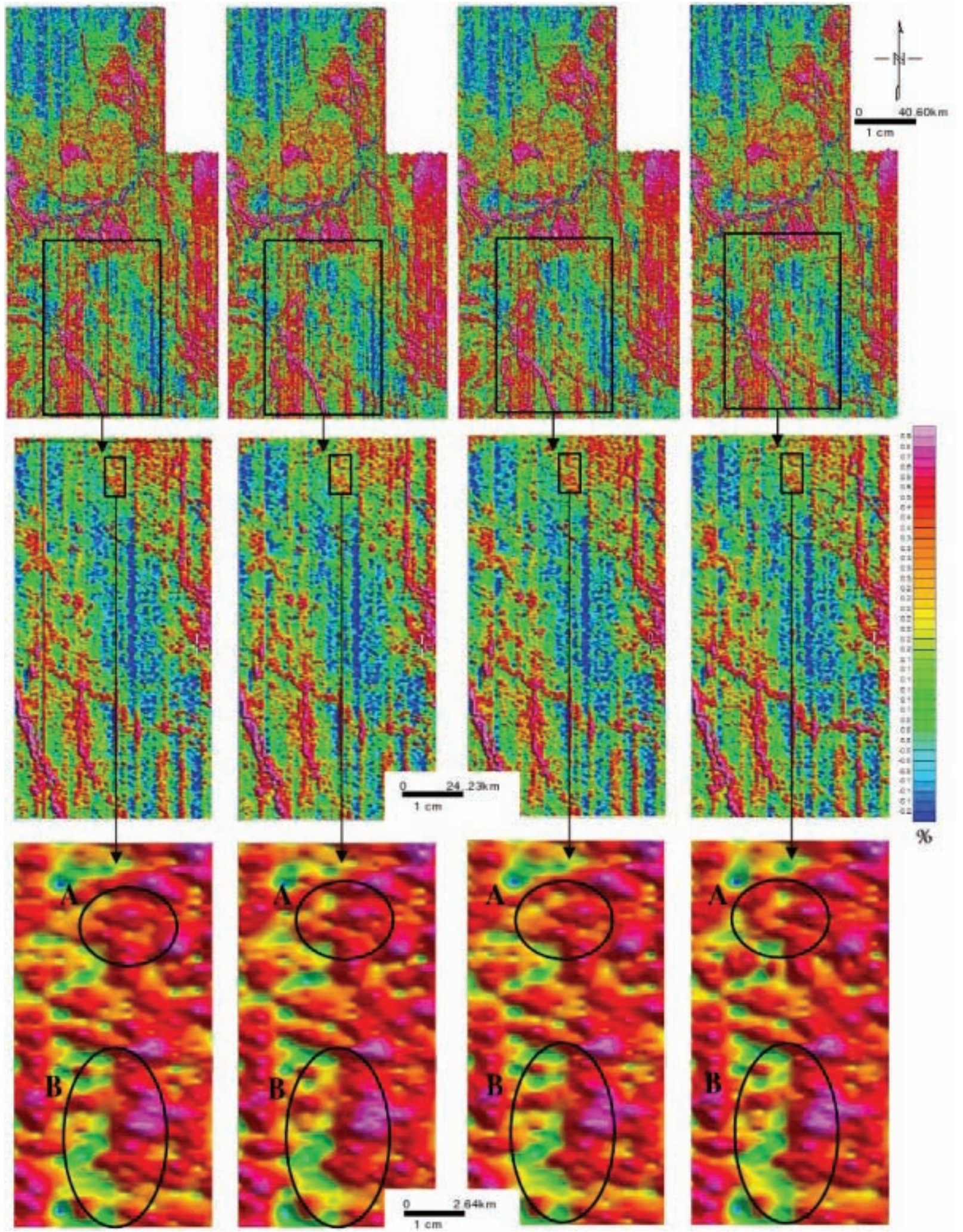

Figura 13 - Mapas de Potássio pós-processados: (a) sem filtragem, (b) filtragem NASVD - 64 componentes, (c) filtragem Passa-Baixa - 3 fiduciais (freqüência de corte) e (d) filtragem MNF - 8 componentes com suas respectivas áreas em detalhamento e ampliação. 


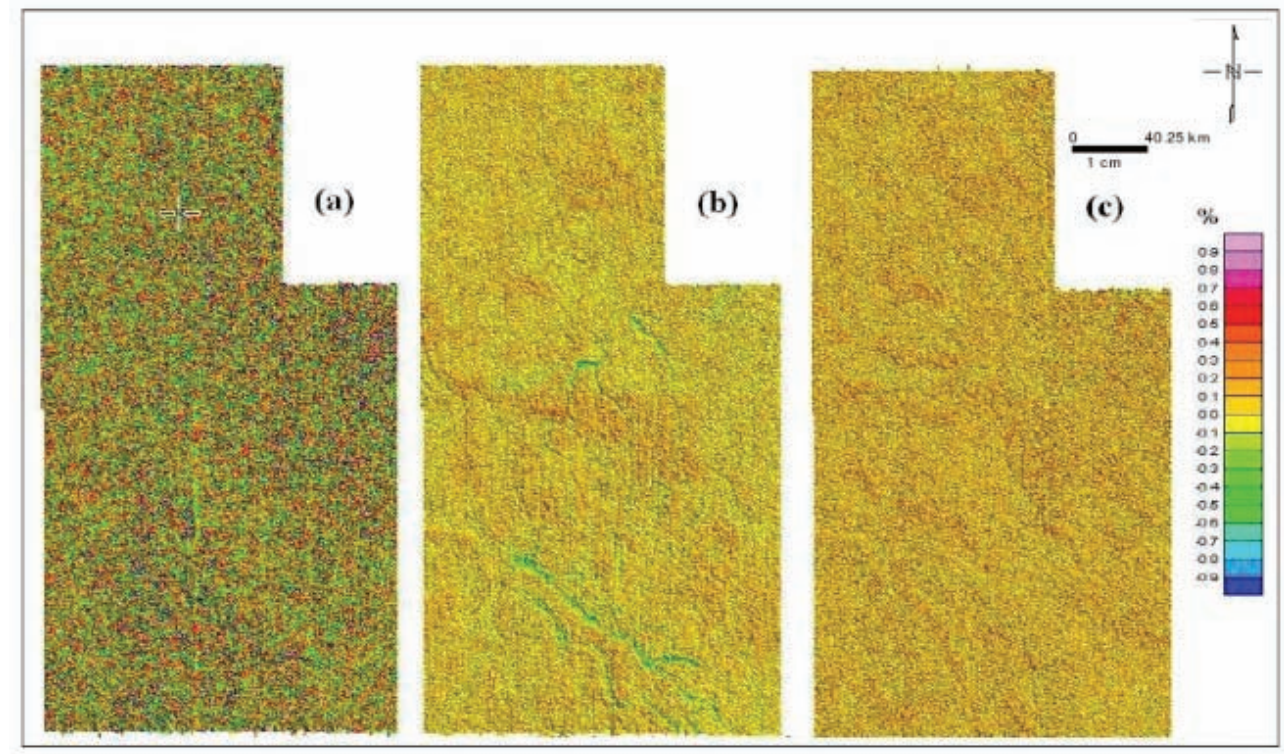

Figura 14 - Mapas das diferenças entre Potássio bruto pós-processado com os filtros Passa-Baixa (a), MNF - 8 componentes (b), NASVD - 64 componentes (c).

\section{REFERÊNCIAS}

CPRM - Serviço Geológico do Brasil. 2008. Projeto Aerogeofísico Província Aurífera do Tapajós. Disponível em:

$<\mathrm{ttp}: / /$ ttp.cprm.gov.br/pub/pdf/tapajos/promin.html>. Acesso em: 27 jul. 2008.

DICKSON B \& TAYLOR G. 1998. Noise reduction of aerial gamma-ray surveys. Exploration Geophysics, 29: 234-329.

DICKSON B \& TAYLOR G. 1999. Why the maximum noise fraction (MNF) method cleans gamma-ray surveys so well. Preview, june 1999, 14-17.

DICKSON B \& TAYLOR G. 2000. Maximum noise fraction method reveals detail in aerial gamma-ray surveys. Exploration Geophysics, 31: 73-77.

GILMORE G \& HEMINGWAY J. 1995. Practical Gamma-Ray Spectrometry. $456 \mathrm{p}$.

GREEN AA, BERMAN M, SWITER P \& CRAIG MD. 1988. A transformation for ordering multispectral data in terms of image quality with implications for noise removal. IEEE Trans. Geosci. and Remote Sensing, GE-26, $65-74$

HOVGAARD J. 1997a. A new processing technique for airborne gammaray spectrometer data (Noise Adjusted Singular Value Decomposition). Am. Nucl. Soc. Sixth Topical Meeting on Emergency Preparedness and Response, 123-127.
HOVGAARD J. 1997b. Airborne Gamma-ray Spectrometry. Statistical Analysis of Airborne Gamma-ray Spectra. Ph.D. thesis, Department of Automation, Technical University of Denmark. $95 \mathrm{p}$.

HOVGAARD J \& GRASTY RL. 1997. Reducing statistical noise in airborne gamma-ray data through spectral component analysis. In: GUBINS AG (Ed.). Proceedings of Exploration 97: Fourth Decennial Conference on Mineral Exploration, 753-764.

IAEA. 2003. Guidelines for Radioelement Mapping using Gamma Ray Spectrometry Data. IAEA TECDOC Series No. 1363, International Atomic Energy Agency, Vienna. $173 \mathrm{p}$.

LASA-FUGRO EP. 1998. Relatório Final do Levantamento e Processamento dos Dados Magnetométricos e Gamaespectrométricos: Projeto Aerogeofísico Província Aurífera do Tapajós - Bloco I e II, volume I texto técnico.

MINTY BRS. 1997. Fundamentals of airborne gamma-ray spectrometry. AGSO Journal of Australian Geology \& Geophysics, 17(2): 39-50.

MINTY BRS. 2000. Reducing Noise in airborne Gamma-ray Spectra. AGSE Preview, december 2000, p. 35-41.

MINTY B \& MCFADDEN P. 1998. Improved NASVD smoothing of airborne gamma-ray spectra. Exploration Geophysics, 29: 516-523.

NOBLE B \& JAMES WD. 1977. Álgebra Linear Aplicada. Ed. PrenticeHall do Brasil Ltda., Rio de Janeiro, capítulo 9. p. 244-283. 


\section{NOTAS SOBRE OS AUTORES}

Francisco de Assis Cavallaro. Graduado em Geofísica pela Universidade de São Paulo (1991). Mestre em Geociências pela Universidade Estadual de Campinas (2008) e doutorando em Geociências pela Universidade de São Paulo. Atua como geofísico na empresa Aerogeophysica Latinoamerica e como desenvolvedor de novas tecnologias na empresa Inforzios Geotecnologies.

Rodrigo de Souza Portugal. Mestre e doutor em Matemática Aplicada pela Universidade Estadual de Campinas (UNICAMP). Em sua tese de doutorado estudou a construção de frentes de onda em situações $2.5 \mathrm{D}$ e suas aplicações em quatro operações de Kirchhoff: modelagem, migração, demigração e demodelagem. Atualmente é professor do Departamento de Geologia e Recursos Naturais (DGRN) da UNICAMP. Seus interesses de pesquisa envolvem propagação de ondas, análises numéricas, imageamento e inversão sísmica e caracterização de reservatórios.

Adalene Moreira Silva. Engenheira Geóloga (1989) graduada pela Universidade Federal de Ouro Preto, Mestre (1992) e Doutora em Geologia (1999) pela Universidade de Brasília. Profissionalmente foi pesquisadora do Serviço Geológico Americano (1997-1999); Professora visitante da Universidade de Brasília (2000-2002); Pesquisadora (05/2002-04/2003) e Professora do Instituto de Geociências da UNICAMP (04/2003-10/2005). Atualmente é Professora do Instituto de Geociências da Universidade de Brasília, e vem ensinando e pesquisando sobre novas técnicas de processamento, interpretação de dados aplicada à exploração mineral, hidrogeologia e ambiente.

Ariathemis Moreno Bizuti. Físico pelo Instituto de Geociências e Ciências Exatas - Universidade Estadual Paulista (2002), Mestre em Física Aplicada (2005) pela Universidade Estadual de Campinas e doutorando em Geociências pela Universidade Estadual de Campinas. Atualmente vem pesquisando novos algoritmos para processamento sísmico e desenvolvimento de novas tecnologias na empresa Inforzios Geotecnologies. 